\title{
Association of Obesity and Hypertension: A Cohort Study in China
}

\author{
Xia Li, ${ }^{1}$ Huiqin Niu $\left(D,{ }^{2}\right.$ XiaoGang Bai, ${ }^{1}$ YuWei Wang, ${ }^{2}$ and Weihua Wang ${ }^{3}$ \\ ${ }^{1}$ Department of Endocrine and Metabolism, Yan'an University Affiliated Hospital, Yan'an, Shaanxi, China \\ ${ }^{2}$ Department of Endocrinology, Cadre Ward, Gansu Provincial People's Hospital, Lanzhou, Gansu, China \\ ${ }^{3}$ Shaanxi Provincial Center for Disease Control and Prevention, Xi'an, Shaanxi, China \\ Correspondence should be addressed to Huiqin Niu; 286435188@qq.com
}

Received 11 July 2021; Accepted 3 November 2021; Published 10 December 2021

Academic Editor: Thereza Maria Magalhães Moreira

Copyright (C) 2021 Xia Li et al. This is an open access article distributed under the Creative Commons Attribution License, which permits unrestricted use, distribution, and reproduction in any medium, provided the original work is properly cited.

\begin{abstract}
The prevalence of general and central obesity has increased rapidly in China for decades, while little is known on obesity-normal weight-central obesity (NWCO) in China. In this study, we aim to depict the trend of the three kinds of obesity and to explore their associations with hypertension in a cohort study in China. We used data from eight waves of the China Health and Nutrition Survey (CHNS) in 1993, 1997, 2000, 2004, 2006, 2009, 2011, and 2015 for analysis. The Cochran-Armitage test was used for trend of the three kinds of obesity or hypertension. Mixed logistic regression was used to explore their relationship. In this study, we found the prevalence of general obesity increased from $20.81 \%$ in 1993 to $50.57 \%$ in 2015 in China, which was from $19.23 \%$ to $56.15 \%$ for central obesity and from $27.20 \%$ to $49.07 \%$ for NWCO, respectively. Males had the highest increase among all the subgroups. The RR for hypertension and general obesity was 3.71 (95\%CI: 3.26-4.22), 3.62 (95\%CI 3.19-4.12) for central obesity, and 1.60 (95\%CI 1.23-2.06) for NWCO after adjusted for age, sex, education, smoking, alcohol drinking, marriage status, urbanicity and income. Both prevalence of obesity and hypertension have increased significantly in China for the two decades. The general obesity was most likely to develop hypertension compared to central or NOCWO in this study.
\end{abstract}

\section{Introduction}

Obesity has become a worldwide public health threat which is the risk factor of many chronic diseases, such as cardiovascular disease, cancer, diabetes, and chronic kidney diseases [1, 2]. There are several factors associated with obesity, such as heredity and disease complications. However, a group of evidence has showed that obesity could be attributed to life styles, for example, dietary pattern, physical activity, smoking, and alcohol drinking [3-5]. With rapid development in economics for decades, the Chinese population has shifted its traditional lifestyles to a more western way, which may be accompanied with more calorie intake in dietary and sedentary lifestyle in workplace and from home [6, 7]. All these factors resulted in an increasing prevalence of obesity. Therefore, the prevalence of obesity in China has increased dramatically from 15.8 to $30.3 \%$ between 1993 and 2011 [8].

Body mass index (BMI), which is defined as weight divided by the square of the height $\left(\mathrm{kg} / \mathrm{m}^{2}\right)$, is the most common indicator to identify general obesity in adults.
Waist circumference (WC), which could consider body fat distribution, is used to evaluate abdominal obesity. It has more significant relationship with disease burden $[9,10]$. Obesity-normal weight-central obesity (NWCO) combined general obesity and abdominal obesity has drawn more attention $[11,12]$. Evidence has showed NWCO was positively associated with systemic inflammation, cardiometabolic dysregulation, and mortality $[12,13]$. Therefore, we use BMI, WC, and NWCO to define general, abdominal obesity, and normal weight with central obesity in this study, respectively.

The optimal predictive obesity indicators for noncommunicable chronic diseases varied among researches. However, these three indicators of obesity negatively associated with various diseases were certain. Growing evidence has shown the positive association of obesity with hypertension. For example, central obesity is a more risk predictor than general obesity for hypertension [14-16]. However, few studies showed the association of NWCO and hypertension, as well as their magnitude. 
Therefore, we describe the secular trend of prevalence of three types of obesity, as depicted by BMI, WC, and NWCO and the prevalence of hypertension. We also explored their relationships, especially the magnitude in a large cohort study of China, to provide perspectives for health interventions on obesity and hypertension.

\section{Methods}

2.1. Study Design and Population. The CHNS was a cohort study on nutrition status and health change among Chinese adults. A multistage, cluster, random sampling method was adopted for the study in China (Liaoning, Shandong, Henan, Jiangsu, Hubei, Hunan, Guizhou, and Guangxi). The study was conducted ten waves from 1989 to 2015. All families and individuals recruited for the first time were invited to participate the follow-ups. Details of the study were published elsewhere $[17,18]$. Height, weight, waist circumference, hip circumference, and blood pressure were measured in the survey from 1993 to 2015. Hence, we used data of CHNS from 1993 to 2015 to explore the associations.

We included participates aged 18-65 in this study. Participants missed key information on BP measurement or hypertension diagnosis record $(N=7540)$, as well as measurement of weight, height, waist circumference, and hip circumference $(N=1611)$, were also excluded. Other missing data were imputed with a multiple imputation method. A total of 23165 participants were involved in the final analysis. The flow chart is shown in Figure 1.

The National Institute of Nutrition and Food Safety (Beijing, China) and the institutional review committees of the University of North Carolina (Chapel Hill, NC, USA) approved the study. Informed consent was obtained from each participant before the survey.

Weight, height, WC, and BP were measured following standardized protocols from the World Health Organization (WHO) [19]. Weight was measured with the participants wearing light clothing on a calibrated beam scale, and height was measured without shoes using a portable stadiometer. BMI was calculated as weight (kilogram) divided by squared height (meter), rounded to the nearest tenth. WC was measured with an inelastic tape to the nearest $0.1 \mathrm{~cm}$ at a midpoint between the bottom of the rib cage and the top of the iliac crest at the end of exhalation. HC was measured at the level of maximal gluteal protrusion using a SECA tape to the nearest $0.1 \mathrm{~cm}$. BP was measured by trained examiners using a mercury sphygmomanometer at three different consecutive times with 3-5 min intervals on one visit. The three readings were averaged as the BP values in our data analysis. All physical examinations were performed at the same location and followed the same protocol at each study visit.

2.2. Definition. General obesity was defined as a BMI $\geq 28 \mathrm{~kg} / \mathrm{m}^{2}$, and central obesity was defined as $\mathrm{WC} \geq 85 \mathrm{~cm}$ for men and $\geq 80 \mathrm{~cm}$ for women, which is recommended by the Working Group on Obesity in China [20]. Therefore, we classified obesity into three types: general obesity (BMI $\geq 28 \mathrm{~kg} / \mathrm{m}^{2}$ ), central obesity (WC $\geq 85 / 80 \mathrm{~cm}$ ), and NWCO $\left(24>\mathrm{BMI} \geq 18 \mathrm{~kg} / \mathrm{m}^{2}\right.$ and waist height ratio $\geq 0.5$ or waist to hip ratio $\geq 0.9 / 0.85$ or waist $>85 / 80 \mathrm{~cm}$ ). Participants with $\mathrm{BMI}<28 \mathrm{~kg} / \mathrm{m}^{2}$ and $\mathrm{WC}<90 / 80 \mathrm{~cm}$ were regarded as normal in this study. Hypertension was defined as a selfreported doctor diagnosis of hypertension, a measured mean systolic blood pressure (SBP) $\geq 140 \mathrm{mmHg}$ or diastolic blood pressure $(\mathrm{DBP}) \geq 90 \mathrm{mmHg}$ [21].

2.3. Covariate. Demographical variables in the study included age (18-35, 36-50, and 51-65 years), sex, marital status, education, ethnicity, residency, and household income. We categorized marital status into currently married and unmarried (single/divorced/widowed). Education was classified into low, middle, and high. Sex (male/female) and residency (urban/rural areas) were also included in the analysis.

Participants who smoked cigarettes or drank alcohol during the previous year were recognized as smokers (including former smokers and current smokers) or drinkers (including former drinkers and current drinkers), separately. In this study, the outcome variables were the prevalence of three types of obesity (general obesity, central obesity, and NWCO) and hypertension.

\subsection{Statistical Analysis. We used mean (standard deviation)} for continuous data normally distributed and median \pm QD for continuous data abnormally distributed and frequency (percentage) for categorical data. The $T$ test or one-way ANOVA and $\chi^{2}$ test were used to compare the difference between groups, respectively.

To explore the relationship between the 3 types of obesity and hypertension, we established a set of models using mixed logistic regression. Model 1 was adjusted for age and sex; model 2 was further adjusted for education, smoking, and alcohol drink; and model 3 was further adjusted for income. All analyses were completed with $R$ 3.4.3. $P<0.05$ was considered statistically significant in this study.

\section{Results}

From 1993 to 2015, 23165 participants with 66744 interviews were enrolled in this study. More female participants than the male were recruited at each survey among the 8 waves. The median age of all the participants increased from 38.00 $(28.00,48.00)$ to $50.00(40.00,58.00)$. The proportion of married participants increased from $80.36 \%$ to $89.97 \%$ from 1993 to 2015, as participants from urban areas and participants with low education level. Proportion of current smokers decreased from $32.11 \%$ to $24.24 \%$ during the 8 waves of survey, so does the proportion of alcohol drinking. The body weight, waist circumference, and hip circumference of participants from 1993 to 2015 increased from $55.00 \mathrm{~kg}$ to $62.80 \mathrm{~kg}, 74.00 \mathrm{~cm}$ to $84.20 \mathrm{~cm}$, and $89.00 \mathrm{~cm}$ to $95.30 \mathrm{~cm}$, respectively, as shown in Table 1 .

The prevalence of general obesity increased from $20.81 \%$ in 1993 to $50.57 \%$ in 2015, in which male were from $17.24 \%$ to $53.32 \%$ and female were from $24.01 \%$ to $48.22 \%$, 


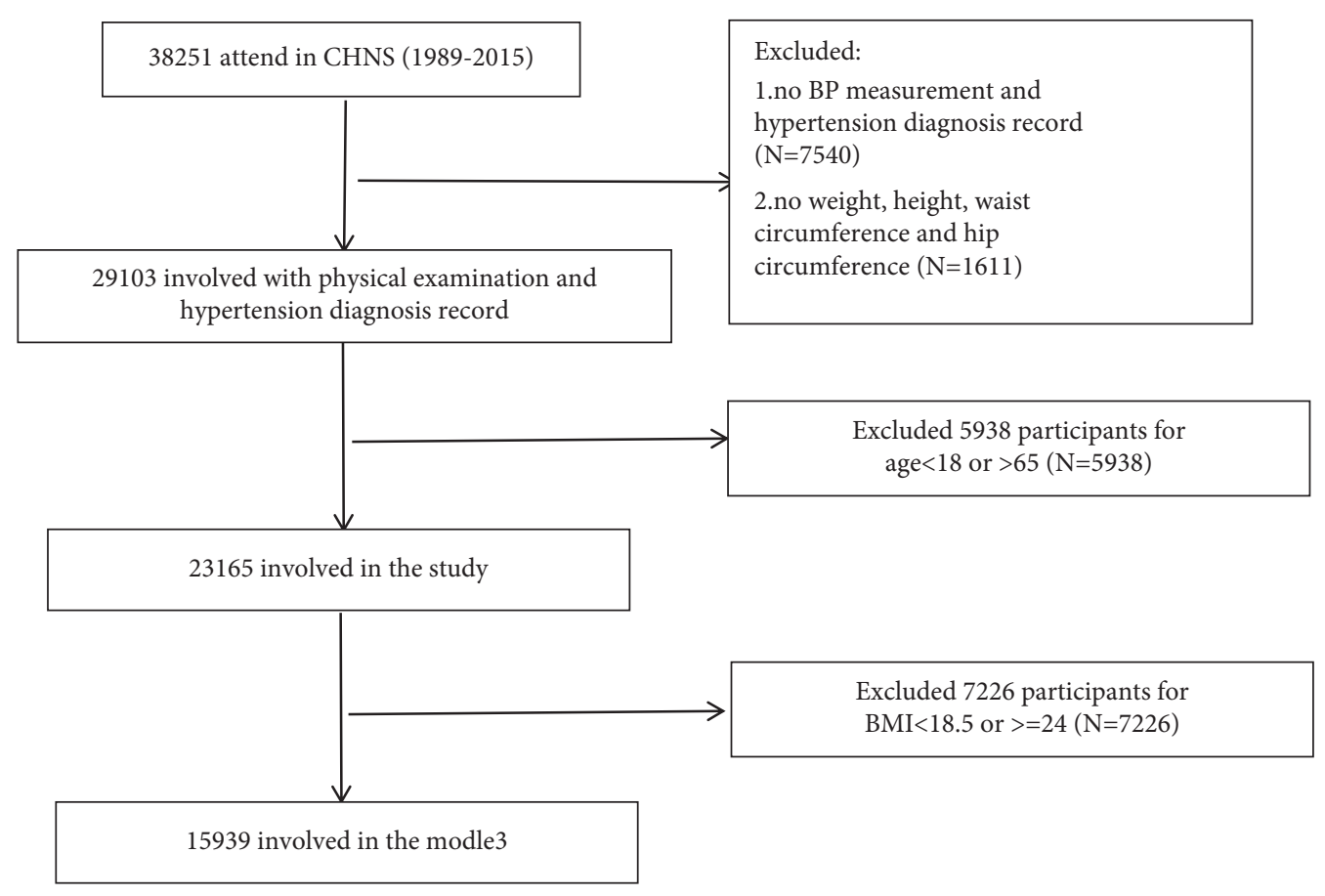

FIGURE 1: Flow chart of participants enrolled in this study measurements.

respectively. The prevalence of central obesity increased from $19.23 \%$ to $56.15 \%$ during the 8 waves of survey, male from $14.80 \%$ to $56.70 \%$ and female from $23.20 \%$ to $55.68 \%$. The prevalence of NWCO was $27.20 \%$ in 1993 and $49.07 \%$ in 2015 , in which $20.39 \%$ was for male and $33.93 \%$ was for female in 1993 and $45.97 \%$ was for male and $51.49 \%$ was for female in 2015. Meanwhile, the general prevalence of hypertension increased from $13.11 \%$ in 1993 to $32.14 \%$ in 2015 , male from $14.86 \%$ to $37.09 \%$ and female from $11.54 \%$ to 27.92\%. Participants aged 51-65 years showed the highest increase from $31.40 \%$ to $45.55 \%$, as shown in Table 2 .

The associations between the 3 types of obesity and hypertension are presented in Table 3 . We adjusted the potential confounders step by step to test the robust of the associations. The RRs was consistent for general obesity and hypertension from model 1 to model 3 (model 1 : RR $=3.46$, 95\%CI 3.24-3.70; model 2: RR = 3.46, 95\%CI 3.24-3.70; and model 3: $\mathrm{RR}=3.71,95 \% \mathrm{CI} 3.26-4.22)$. The RR for central obesity and hypertension was 2.89 (95\%CI 2.71-3.08) in model 1 and 3.62 (95\%CI 3.19-4.12) in model 3. The association between NWCO and hypertension was 1.60 (95\% CI 1.23-2.06) after we adjusted for age, sex, education, smoking, alcohol drink, marriage status, urbanicity, and income.

\section{Discussion}

Our study demonstrated that the prevalence of general obesity, central obesity, and NWCO increased significantly in China from 1993 to 2015 . Attention should be drawn that all of the highest increases of the three types of obesity were among the male in this study. The prevalence of hypertension increased nearly $20 \%$ among Chinese adults from
1993 to 2015. Participants with general and central obesity had risk of incident hypertension, whereas those who were NWCO also had risk to develop hypertension.

The study showed that the obesity increased significantly in China during the two decades. The prevalence of general obesity increased from $20.81 \%$ to $50.57 \%$ in China, which is in line with previous studies $[22,23]$. Male had the highest increase compared to their counterparts in our study. This finding is similar to the study in Korean men, whose general obesity increased from $22.1 \%$ to $27.5 \%$ in 10 years [24]. The unhealthy and irregular diets with high calories and socializing with alcohol binge plus with sedentary lifestyle may attribute to the high increase of general obesity, especially among male residents $[25,26]$. The high increase of obesity among the old-age group also needed to be noticed, which could possibly place a heavy disease burden related to obesity in the future. We observed the same trend in central obesity, and central obesity had the highest increase among the three types of obesity, which is similar to other East Asian countries $[24,27]$. What is worthy of attention is that the prevalence of NWCO also increased from $20.39 \%$ in 1993 to $45.97 \%$ in 2015, which is more than doubled. Seldom studies have the report; however, NOWCO is often ignored, but is a risk to heath. Some studies found that NWCO was associated with hypertension, diabetes, insulin resistance, low HDL, and high TG $[28,29]$. Public health policy should be made to prevent and control NOWCO besides general or central obesity.

Meanwhile, we found in this study that the prevalence of hypertension increased more than doubled from $13.11 \%$ in 1993 to $32.14 \%$ in 2015. Other studies in China also revealed the high increase of prevalence of hypertension $[30,31]$. However, it is estimated that the prevalence would grow $55 \%$ 


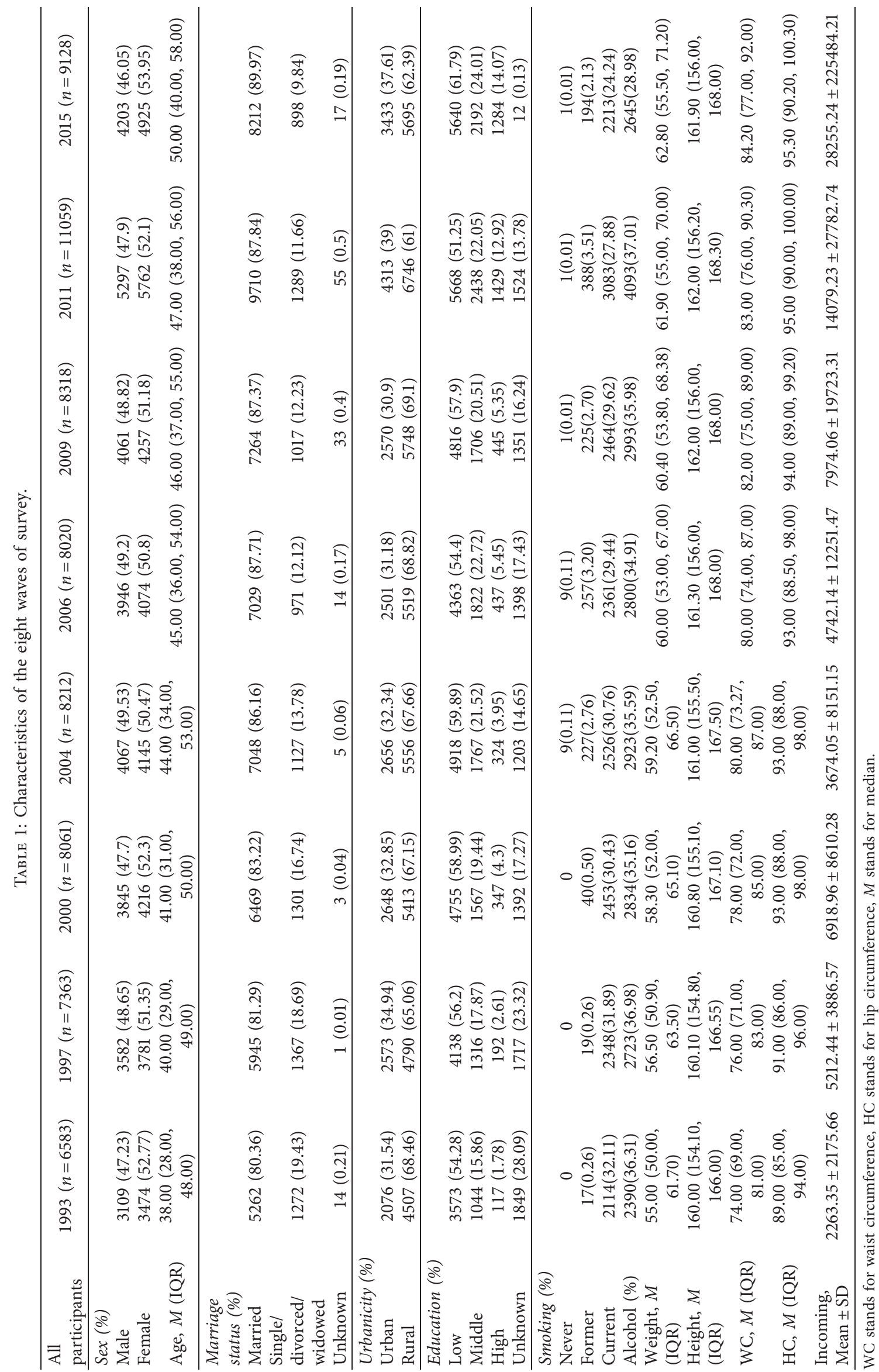


TABLE 2: The prevalence of three types of obesity and hypertension.

\begin{tabular}{|c|c|c|c|c|c|c|c|c|}
\hline Outcomes (\%) & $\begin{array}{c}1993 \\
(n=6583)\end{array}$ & $\begin{array}{c}1997 \\
(n=7363)\end{array}$ & $\begin{array}{c}2000 \\
(n=8061)\end{array}$ & $\begin{array}{c}2004 \\
(n=8212)\end{array}$ & $\begin{array}{c}2006 \\
(n=8020)\end{array}$ & $\begin{array}{c}2009 \\
(n=8318)\end{array}$ & $\begin{array}{c}2011 \\
(n=11059)\end{array}$ & $\begin{array}{c}2015 \\
(n=9128)\end{array}$ \\
\hline $\begin{array}{l}\text { General } \\
\text { besity }\end{array}$ & $1370(20.81)$ & $925(26.14)$ & $2592(32.15)$ & $2889(35.18)$ & $965(36.97)$ & $3333(40.07)$ & $5001(45.22)$ & $4616(50.57)$ \\
\hline & $536(17.24)$ & $828(23.12)$ & $1170(30.43)$ & $1374(33.78)$ & $445(36.62)$ & $1677(41.30)$ & $2485(46.91)$ & $2241(53.32)$ \\
\hline & & 1097 & & & & & & \\
\hline ge18-35 years & 3 & & 602 & & & & & \\
\hline & & & & & & & & \\
\hline & 3 & & & 1060 & & & & \\
\hline S & $266(19.23)$ & $815(2465)$ & $2535(31.45)$ & $3063(37.30)$ & $153(20$ 31) & $3793(45.60)$ & $5657(51.15)$ & \\
\hline & & 702 (21) & & 1423 (34.99) & $1476(27$ & & & \\
\hline & & & & & & & & \\
\hline 35 years & & & & & & & & \\
\hline & & & & & & & & \\
\hline ge 5 & 445( & & & 1224 & & & & \\
\hline & 1256 & & & & & & & \\
\hline & & & & & & & & \\
\hline & & & & & & & & \\
\hline & & & & & & & & \\
\hline & & & & & & & & \\
\hline & & & & & & & & \\
\hline & & & & & & & & \\
\hline & & & & & & & & \\
\hline & & & & $675(16.28)$ & & & $1209(20.98)$ & $1375(27.92)$ \\
\hline Age $18-35$ years & $140(4.92)$ & & $163(5.77)$ & $141(6.22)$ & & & & $147(9.57)$ \\
\hline & $290(12.30)$ & & & & & & & $817(25.01)$ \\
\hline Age $51-65$ years & $433(31.40)$ & $558(33.84)$ & $722(36.06)$ & $876(33.95)$ & $882(31.60)$ & $1226(38.36)$ & $1721(37.81)$ & $1970(45.55)$ \\
\hline
\end{tabular}

WC stands for waist circumference; NWCO stands for normal weight with central obesity.

TABLE 3: Association between the 3 types of obesity and hypertension.

\begin{tabular}{lccr}
\hline RR $(95 \%$ CI $)$ & General obesity & Central obesity & NWCO \\
\hline Model 1 & $3.46(3.24-3.70)$ & $2.89(2.71-3.08)$ & $1.42(1.30-1.56)$ \\
Model 2 & $3.31(2.97-3.70)$ & $3.12(2.81-3.46)$ & $1.48(1.28-1.71)$ \\
Model 3 & $3.71(3.26-4.22)$ & $3.62(3.19-4.12)$ & $1.60(1.23-2.06)$ \\
\hline
\end{tabular}

Model 1: adjusted for age and sex; model 2: further adjusted for education (tertiles), smoking (tertiles), alcohol drink, and marriage status; model 3: further adjusted for urbanicity and income (continuous).

by year 2050 if no interventions on hypertension control are taken [32]. That is a huge challenge for China, economically and socially. It is suggested that further public policies and measures on hypertension should be implemented.

We also investigated the relationship between the three types of obesity and hypertension in this study. The association between obesity and hypertension has been widely reported [33-35]. However, few studies compared their different associations of the three types of obesity in one study, particularly the association with NWCO. The results in this study have demonstrated that the risk of incident hypertension was general obesity, followed by central obesity and NWCO. Few studies on this topic were available [8]. Due to the different definition of obesity, the order of RR is different from the previous one. However, attention should be paid to NWCO besides the other two types of obesity.

There are several limitations in this study. First, the latest released data of CHNS are wave of 2015; thus, we could not show the updated results in this study. Second, some time-dependent variables could not control in the models since covariates adjusted in the models were investigated in baseline survey. Third, the trends in this study were only China-representative, no subnational level or provincial level could conclude in this study. Last, the proportion of senior citizens was large with the wave, so more young ones should recruit in the following waves of the survey.

In conclusion, we found that both prevalence of obesity and hypertension have increased significantly in the two decades. The general obesity was most likely to develop hypertension compared to central or NOCWO in this study.

\section{Data Availability}

Data can be found on the Internet via searching for CHNS.

\section{Conflicts of Interest}

The authors declare no conflicts of interest. 


\section{Acknowledgments}

The authors thank all the staff members in their institution.

\section{References}

[1] M. Ng, T. Fleming, M. Robinson, B. Thomson, N. Graetz, and C. Margono, "Global, regional, and national prevalence of overweight and obesity in children and adults during 1980-2013: a systematic analysis for the Global Burden of Disease Study," Lancet, vol. 384, pp. 766-781, 2014.

[2] M. M. Finucane, G. A. Stevens, M. J. Cowan et al., "National, regional, and global trends in body-mass index since 1980: systematic analysis of health examination surveys and epidemiological studies with 960 country-years and 9.1 million participants," The Lancet, vol. 377, no. 9765, pp. 557-567, 2011.

[3] S. K. Tanamas, J. E. Shaw, K. Backholer, D. J. Magliano, and A. Peeters, "Twelve-year weight change, waist circumference change and incident obesity: the Australian diabetes, obesity and lifestyle study," Obesity, vol. 22, no. 6, pp. 1538-1545, 2014.

[4] F. Zhai, H. Wang, S. Du, Y. He, Z. Wang, and K. Ge, "Prospective study on nutrition transition in China," Nutrition Reviews, vol. 67, no. 1, Article ID S56eS61, 2009.

[5] S. W. Ng, E. C. Norton, and B. M. Popkin, "Why have physical activity levels declined among Chinese adults? Findings from the 1991-2006 China health and nutrition surveys," Social Science \& Medicine, vol. 68, no. 7, pp. 1305-1314, 2009.

[6] B. M. Popkin, S. Horton, S. Kim, A. Mahal, and J. Shuigao, "Trends in diet, nutritional status, and diet-related noncommunicable diseases in China and India: the economic costs of the nutrition transition," Nutrition Reviews, vol. 59, no. 12 , pp. 379-390, 2001.

[7] H. Wang, S. Du, F. Zhai, and B. M. Popkin, "Trends in the distribution of body mass index among Chinese adults, aged 20-45 years (1989-2000)," International Journal of Obesity, vol. 31, no. 2, pp. 272-278, 2007.

[8] C. Shen, Z. Zhou, S. Lai et al., "Urban-rural-specific trend in prevalence of general and central obesity, and association with hypertension in Chinese adults, aged 18-65 years," BMC Public Health, vol. 19, no. 1, p. 661, 2019.

[9] J. H. Park, I.-C. Cho, Y. S. Kim, S. K. Kim, S. K. Min, and S. S. Kye, "Body mass index, waist-to-hip ratio, and metabolic syndrome as predictors of middle-aged men's health," Korean Journal of Urology, vol. 56, no. 5, pp. 386-392, 2015.

[10] J. Niu and D.-C. Seo, "Central obesity and hypertension in Chinese adults: a 12-year longitudinal examination," Preventive Medicine, vol. 62, pp. 113-118, 2014.

[11] E. Oliveros, V. K. Somers, O. Sochor, K. Goel, and F. LopezJimenez, "The concept of normal weight obesity," Progress in Cardiovascular Diseases, vol. 56, no. 4, pp. 426-433, 2014.

[12] A. Romero-Corral, V. K. Somers, J. Sierra-Johnson et al., "Normal weight obesity: a risk factor for cardiometabolic dysregulation and cardiovascular mortality," European Heart Journal, vol. 31, no. 6, pp. 737-746, 2009.

[13] T. Coutinho, K. Goel, D. Corrêa de Sá et al., "Combining body mass index with measures of central obesity in the assessment of mortality in subjects with coronary disease," Journal of the American College of Cardiology, vol. 61, no. 5, pp. 553-560, 2013.

[14] M. Zhang, Y. Zhao, G. Wang et al., "Body mass index and waist circumference combined predicts obesity-related hypertension better than either alone in a rural Chinese population," Scientific Reports, vol. 6, no. 1, p. 31935, 2016.
[15] X. Zhang, X.-O. Shu, Y.-T. Gao, G. Yang, H. Li, and W. Zheng, "General and abdominal adiposity and risk of stroke in Chinese women," Stroke, vol. 40, no. 4, pp. 1098-1104, 2009.

[16] T. Du, X. Sun, P. Yin, R. Huo, C. Ni, and X. Yu, "Increasing trends in central obesity among Chinese adults with normal body mass index, 1993-2009," BMC Public Health, vol. 13, no. 1, p. 327, 2013.

[17] B. M. Popkin, S. Du, F. Zhai, and B. Zhang, "Cohort profile: the China health and nutrition survey-monitoring and understanding socio-economic and health change in China, 1989-2011," International Journal of Epidemiology, vol. 39, no. 6, pp. 1435-1440, 2010.

[18] B. Zhang, F. Y. Zhai, S. F. Du, and B. M. Popkin, "The China health and nutrition survey, 1989-2011," Obesity Reviews, vol. 15, no. 1, pp. 2-7, 2014.

[19] WHO, IASO, and IOTF, The Asia-Pacific Perspective: Redefining Obesity and Its Treatment, International Diabetes Institute, Melbourne, Australia, 2000.

[20] B. F. Zhou, "Cooperative meta-analysis group of the working group on obesity in C. Predictive values of body mass index and waist circumference for risk factors of certain related diseases in Chinese adultse study on optimal cut-off points of body mass index and waist circumference in Chinese adults," Biomedical and Environmental Sciences, vol. 15, Article ID 83e96, 2002.

[21] A. V. Chobanian, G. L. Bakris, H. R. Black, W. C. Cushman, L. A. Green, and J. L. IzzoJr, "The seventh report of the joint national committee on prevention, detection, evaluation, and treatment of high blood pressure: the JNC 7 report," JAMA, vol. 289, no. 19, pp. 2560-2572, 2003.

[22] Y.-J. Mi, B. Zhang, H.-J. Wang et al., "Prevalence and secular trends in obesity among Chinese adults, 1991-2011," American Journal of Preventive Medicine, vol. 49, no. 5, pp. 661-669, 2015.

[23] B. Xi, Y. Liang, T. He et al., "Secular trends in the prevalence of general and abdominal obesity among Chinese adults, 1993-2009," Obesity Reviews, vol. 13, no. 3, pp. 287-296, 2012.

[24] S. Yoo, H.-J. Cho, and Y.-H. Khang, "General and abdominal obesity in South Korea, 1998-2007: gender and socioeconomic differences," Preventive Medicine, vol. 51, no. 6, pp. $460-465,2010$.

[25] P. Koh-Banerjee, N.-F. Chu, D. Spiegelman et al., "Prospective study of the association of changes in dietary intake, physical activity, alcohol consumption, and smoking with 9-y gain in waist circumference among 16587 US men," American Journal of Clinical Nutrition, vol. 78, no. 4, pp. 719-727, 2003.

[26] Y. Sakurai, T. Umeda, K. Shinchi et al., "Relation of total and beverage-specific alcohol intake to body mass index and waist-tohip ratio: a study of self-defense officials in Japan," European Journal of Epidemiology, vol. 13, no. 8, pp. 893-898, 1997.

[27] B. Matsuura, H. Nunoi, T. Miyake, Y. Hiasa, and M. Onji, "Obesity and gastrointestinal liver disorders in Japan," Journal of Gastroenterology and Hepatology, vol. 28, no. 4, Article ID 48e53, 2013.

[28] B. Francilene, Madeira, A. A. Silva, M. Z. Goldani, G. Kac, and V. C. Cardoso, "Normal weight obesity is associated with metabolic syndrome and insulin resistance in young adults from a middle-income country," PLoS One, vol. 8, Article ID e60673, 2013.

[29] P. Song, X. Li, Y. Bu et al., "Temporal trends in normal weight central obesity and its associations with cardiometabolic risk among Chinese adults," Scientific Reports, vol. 9, no. 1, p. 5411, 2019.

[30] M. Bao and L. Wang, "The longitudinal trend of hypertension prevalence in Chinese adults from 1959 to 2018: a systematic 
review and meta-analysis," Annals of Palliative Medicine, vol. 9, no. 5, pp. 2485-2497, 2020.

[31] L. Zheng, Y. Dai, P. Fu et al., "Secular trends of hypertension prevalence based on 2017 ACC/AHA and 2018 Chinese hypertension guidelines: results from CHNS data (1991-2015)," Journal of Clinical Hypertension, vol. 23, no. 1, pp. 28-34, 2021.

[32] N. Sudharsanan and P. Geldsetzer, "Impact of coming demographic changes on the number of adults in need of care for hypertension in Brazil, China, India, Indonesia, Mexico, and South Africa," Hypertension, vol. 73, no. 4, pp. 770-776, 2019.

[33] Y. Wang, J. Mi, X.-y. Shan, Q. J. Wang, and K.-y. Ge, "Is China facing an obesity epidemic and the consequences? The trends in obesity and chronic disease in China," International Journal of Obesity, vol. 31, no. 1, pp. 177-188, 2007.

[34] M. Zhang, Y. Zhao, H. Sun et al., "Effect of dynamic change in body mass index on the risk of hypertension: results from the rural Chinese cohort study," International Journal of Cardiology, vol. 238, pp. 117-122, 2017.

[35] M. Mohammadi and M. Mirzaei, "Population-attributable fraction of hypertension associated with obesity, abdominal obesity, and the joint effect of both in the central provinces of Iran," Journal of epidemiology and global health, vol. 7, no. 1, pp. 71-79, 2017. 